\title{
Characterization and Estimation of Length Biased Weighted Generalized Uniform Distribution
}

\author{
A. A. Rather $^{1^{*}}$ and C. Subramanian ${ }^{2}$ \\ ${ }^{1,2}$ Department of Statistics, Annamalai University, Annamalai Nagar, Tamil Nadu, India \\ *Corresponding Author: aafaq7741@gmail.com
}

Available online at: www.isroset.org

Received: 23/Sept/2018, Accepted:13/Oct/2018, Online: 31/Oct/2018

\begin{abstract}
In this paper, we proposed a new probability model called as the length biased weighted generalized uniform distribution (LBWGUD) and discussed its various statistical properties. The probability density function, moments, hazard rate function, reverse hazard rate function and survival function have been derived. The maximum likelihood method has been used to estimate the parameter and their asymptotics have been discussed.
\end{abstract}

Keywords: Weighted distribution, Moments, Reliability Analysis, Order Statistics, Maximum Likelihood Estimation, Fisher's information matrix.

\section{INTRODUCTION}

The weighted distributions are applied in various research areas related to biomedicine, reliability, ecology and branching processes. In many applied sciences like engineering, medicine, behavioural science, finance, insurance and others, it is very crucial to modelling and analyzing lifetime data. For modelling this type of lifetime data, a number of continuous distributions are for modelling like weibull, lindley, exponential, lognormal and gamma. If the weight function considers the length only in units, then the weighted distribution reduces to length biased weighted distribution. Generally, the size-biased distribution is when the sampling mechanism selects the units with probability which is proportional to some measure of the unit size. The concept of weighted distribution was given by Fisher (1934). Later it was modified by Rao (1965) in a unified manner, where by weighted distributions many situations can be solved. Further, Van Deusen (1986) fitting data related to diameter at breast height $(\mathrm{DBH})$ arising from horizontal point sampling (HPS) in a size biased distribution. Also Lappi and Bailey (1987), used size biased distributions for analysing HPS diameter increment data. Para and Jan (2018) introduced the weighted pareto type-II distribution as a new model for handling medical science data and studied its statistical properties and applications. Recently, Rather et al (2018) obtained a new size biased Ailamujia distribution with applications in engineering and medical science which shows more flexibility than classical distributions.
A probability distribution can be characterized through various methods. Generalized uniform distribution is characterized through the conditional expectation of lower record values. Ali et al. (2007), study a new property exponentiated generalized uniform distribution. Bhatt (2014), discussed characterization of generalized uniform distribution through expectation. Khan and khan (2017), obtained the characterization of generalized uniform distribution based on lower record values.

Consider the generalized uniform distribution with probability density function (pdf) as

$$
\begin{gathered}
\quad g(x ; \alpha, \beta)=\left(\frac{\alpha+1}{\beta}\right)\left(\frac{x}{\beta}\right)^{\alpha} \\
0<x<\beta,-1<\alpha
\end{gathered}
$$

its cumulative distribution function (cdf) is

$$
G(x ; \alpha, \beta)=\left(\frac{x}{\beta}\right)^{\alpha+1}
$$

And its mean is given by

$$
E(x)=\frac{\beta(\alpha+1)}{(\alpha+2)}
$$

\section{LENGTH BIASED WEIGHTED GENERALIZED UNIFORM DISTRIBUTION (LBWGUD)}

A non-negative random variable $X$ is said to have weighted distribution, if the pdf of weighted random variable $X_{w}$ is given by 


$$
f_{w}(x)=\frac{w(x) g(x)}{E(w(x))}, x>0
$$

where $w(x)$ be a non-negative weight function and $E(w(x))=\int w(x) f(x) d x<\infty$.

For different weighted models, we have different choice of the weight function $w(x)$. When $w(x)=x^{c}$, the resulting distribution is termed as weighted distribution. In this paper, we have to find the length biased version of weighted generalized uniform distribution, so we will take $\mathrm{c}=1$ in weights $x^{c}$, in order to get the length biased weighted generalized uniform distribution (LBWGUD) and its pdf is given by:

$$
f_{l}(x)=\frac{x f(x)}{E(x)}, x>0
$$

By applying the values of (1) and (2) in equation (3), we will get the pdf of length biased weighted generalized uniform distribution.

$$
f_{l}(x ; \alpha, \beta)=\frac{(\alpha+2) x^{\alpha+1}}{\beta^{\alpha+2}}
$$

and the corresponding cdf of length biased weighted generalized uniform distribution can be obtained as

$$
\begin{gathered}
F_{l}(x ; \alpha, \beta)=\int_{0}^{x} f_{l}(x) d x \\
=\int_{0}^{x} \frac{(\alpha+2) x^{\alpha+1}}{\beta^{\alpha+2}} d x
\end{gathered}
$$

After simplification, we will get the required cdf of LBWGUD as

$$
F_{l}(x ; \alpha, \beta)=\left(\frac{x}{\beta}\right)^{\alpha+2}
$$

where $\alpha$ is the shape parameter and $\beta$ is scale parameter.



Fig.1: pdf plot of Length biased Generalized Uniform distribution

\section{RELIABILITY ANALYSIS}

In this section, we will present the reliability function, hazard rate function and reverse hazard rate function for the length biased weighted generalized uniform distribution. The probability that a system survives beyond a specified time is known as reliability function or survivor or survival function and is given by

$$
\begin{aligned}
& S(x)=1-F(x) \\
& S(x)=1-\left(\frac{x}{\beta}\right)^{\alpha+2}
\end{aligned}
$$

The hazard function is also called as hazard rate or failure rate and is given by

$$
\begin{aligned}
& h(x)=\frac{f(x)}{1-F(x)} \\
& h(x)=\frac{(\alpha+2) x^{\alpha+1}}{\beta^{\alpha+2}-x^{\alpha+2}}
\end{aligned}
$$

and the reverse hazard function for the length biased weighted generalized uniform distribution is given by

$$
\begin{gathered}
h_{r}(x)=\frac{f(x)}{F(x)} \\
h_{r}(x)=\frac{(\alpha+2)}{x}
\end{gathered}
$$

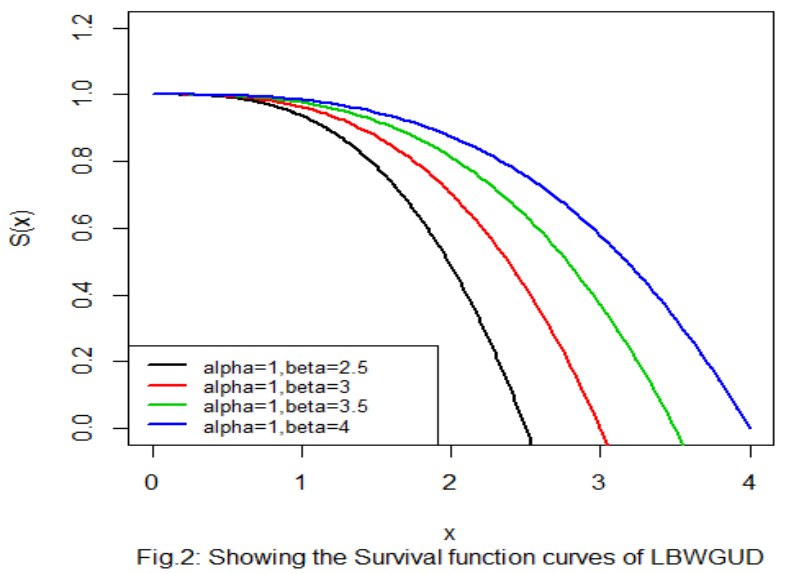

\section{MOMENTS}

Let $X$ denotes the random variable of length biased weighted generalized uniform distribution with parameter $\alpha$ and $\beta$, then the r-th order moment $E\left(X^{r}\right)$ of length biased 
weighted generalized uniform distribution can be obtained as

$$
\begin{aligned}
E\left(X^{r}\right) & =\mu_{r}{ }^{\prime}=\int_{0}^{\infty} x^{r} f_{l}(x ; \theta) d x \\
& =\int_{0}^{\beta} \frac{x^{r}(\alpha+2) x^{\alpha+1}}{\beta^{\alpha+2}} d x \\
& =\frac{(\alpha+2)}{\beta^{\alpha+2}} \int_{0}^{\beta} x^{\alpha+r+1} d x \\
& =\frac{(\alpha+2) \beta^{\alpha+r+2}}{(\alpha+r+2) \beta^{\alpha+2}}
\end{aligned}
$$

Put $r=1,2,3,4$ we will obtain the first four moments

$$
\begin{aligned}
& \text { Mean }=\mu_{1}^{\prime}=\frac{\beta(\alpha+2)}{(\alpha+3)} \\
& \text { Also } \mu_{2}^{\prime}=\frac{\beta^{2}(\alpha+2)}{(\alpha+4)} \\
& \text { Variance }=\frac{\beta^{2}(\alpha+2)}{(\alpha+4)(\alpha+3)^{2}}
\end{aligned}
$$

Standard Deviation $(\sigma)=\frac{\beta}{(\alpha+3)} \sqrt{\frac{(\alpha+2)}{(\alpha+4)}}$

$$
M_{x}(t)=E\left(e^{t x}\right)=\int_{0}^{\beta} e^{t x} f_{l}(x ; \alpha, \beta) d x
$$

Using Taylor's series

$$
\begin{aligned}
M_{x}(t)=E\left(e^{t x}\right) & =\int_{0}^{\beta}\left(1+t x+\frac{(t x)^{2}}{2 !}+\cdots\right) f(x) d x \\
& =\int_{0}^{\beta} \sum_{j=0}^{\infty} \frac{t^{j}}{j !} x^{j} f(x) d x \\
& =\sum_{j=0}^{\infty} \frac{t^{j}}{j !} \mu_{j}^{\prime} \\
\Rightarrow M_{x}(t) & =(\alpha+2) \sum_{j=0}^{\infty} \frac{t^{j} \beta^{j}}{j !(\alpha+j+2)}
\end{aligned}
$$

Similarly, the characteristic function of LBWGUD can be obtained in a similar way

$$
\begin{aligned}
& \varphi_{x}(t)=M_{x}(i t) \\
\Rightarrow & M_{x}(t)=(\alpha+2) \sum_{j=0}^{\infty} \frac{t^{j} \beta^{j}}{j !(\alpha+j+2)}
\end{aligned}
$$

\section{MAXIMUM LIKELIHOOD ESTIMATORS AND FISHER'S INFORMATION MATRIX}

In this section, we will use maximum likelihood method for estimating the parameters of length biased weighted generalized uniform distribution. Let $X_{1}, X_{2}, \ldots, X_{n}$ denotes the random sample of size $n$ from the length biased weighted generalized uniform distribution. Then the likelihood function is given by

Coefficient of Variation (C.V.) $=\frac{\sigma}{\mu_{1}^{\prime}}=\frac{1}{(\alpha+2)} \sqrt{\frac{(\alpha+2)}{(\alpha+4)}}$

$$
L(\alpha, \beta)=\frac{(\alpha+2)^{n}}{\beta^{n(\alpha+2)}} \prod_{i=1}^{n} x_{i}^{\alpha+1}
$$

Coefficient of Dispersion $(\gamma)=\frac{\sigma^{2}}{\mu_{1}^{\prime}}=\frac{\beta}{(\alpha+3)(\alpha+4)}$

\section{MOMENT GENERATING FUNCTION AND CHARACTERISTIC FUNCTION OF LBWGUD}

Let $X$ have a length biased weighted generalized uniform distribution, then the moment generating function of $X$ is given by
The log-likelihood function comes out to be

$$
\log L(\alpha, \beta)=n \log (\alpha+2)-n(\alpha+2) \log \beta+(\alpha+1) \sum_{i=1}^{n} \log x_{i}
$$

Therefore the maximum likelihood estimator of $\alpha, \beta$ which maximize equation (7), must satisfy the following normal equations given by 


$$
\frac{\partial}{\partial \alpha} \log L(\alpha, \beta)=\frac{n}{\alpha+2}-n \log \beta+\sum_{i=1}^{n} \log x_{i}=0 E\left(\frac{\partial^{2} \log L}{\partial \beta^{2}}\right)=\frac{n(\alpha+2)}{\beta^{2}}, \text { (8) } E\left(\frac{\partial^{2} \log L}{\partial \beta \partial \alpha}\right)=-\frac{n}{\beta}
$$$$
\frac{\partial}{\partial \beta} \log L(\alpha, \beta)=\frac{-n(\alpha+2)}{\beta}=0
$$

$\lambda$ being unknown, we estimate $I(\mathcal{G})(\lambda)$ by $I^{-1}(\hat{\lambda})$ and can use this to obtain asymptotic confidence intervals for $\alpha$ and $\beta$.

From (8), we get

$n-n \alpha \log \beta-2 n \log \beta+\alpha \sum_{i=1}^{n} \log x_{i}+2 \sum_{i=1}^{n} \log x_{i}=0$

$$
\Rightarrow \hat{\alpha}=\left(\frac{n-2 n \log \beta+2 \sum_{i=1}^{n} \log x_{i}}{n \log \beta-\sum_{i=1}^{n} \log x_{i}}\right)
$$

And from (9), we get

$$
\hat{\beta}=\infty \text {, which is an absured result. }
$$

Here we apply inspection method. Let us consider the nordered samples $X_{(1)}, X_{(2)}, \ldots ., X_{(n)}$, then

$$
\begin{aligned}
& \quad 0 \leq X_{(1)} \leq X_{(2)} \leq \ldots \leq X_{(n)} \leq \beta \\
& \Rightarrow \beta \geq X_{(n)}
\end{aligned}
$$

Therefore, MLE of $\beta=X_{(n)}=$ the largest sample observations.

In order to obtain confidence interval we use the asymptotic normality results. Suppose that if $\hat{\lambda}=(\hat{\alpha}, \hat{\beta})$ denotes the MLE of $\lambda=(\alpha, \beta)$, then

$$
\sqrt{n}(\hat{\lambda}-\lambda) \rightarrow N_{2}\left(O, I^{-1}(\lambda)\right)
$$

Where $I^{-1}(\lambda)$ is the Fisher's Information Matrix and is given by

$$
I(\lambda)=-\frac{1}{n}\left[\begin{array}{ll}
E\left(\frac{\partial^{2} \log L}{\partial \alpha^{2}}\right) & \left.E\left(\frac{\partial^{2} \log L}{\partial \alpha \partial \beta}\right)\right] \\
E\left(\frac{\partial^{2} \log L}{\partial \beta \partial \alpha}\right) & E\left(\frac{\partial^{2} \log L}{\partial \beta^{2}}\right)
\end{array}\right]
$$

Where

$$
E\left(\frac{\partial^{2} \log L}{\partial \alpha^{2}}\right)=-\frac{n}{(\alpha+2)^{2}}, \quad E\left(\frac{\partial^{2} \log L}{\partial \alpha \partial \beta}\right)=-\frac{n}{\beta}
$$

\section{ORDER STATISTICS}

Order statistics plays an important role in many theoretical as well as practical fields. Let $X_{(1)}, X_{(2)}, \ldots, X_{(n)}$ be the order statistics of a random sample $X_{1}, X_{2}, \ldots, X_{n}$ from the continuous population with probability density function $f_{x}(x)$ and cumulative density function $F_{x}(x)$, then the pdf of $r^{\text {th }}$ order statistics $X_{(r)}$ is given by

$$
f_{X(r)}(x)=\frac{n !}{(r-1) !(n-r) !} f_{X}(x)\left[F_{X}(x)\right]^{r-1}\left[1-F_{X}(x)\right]^{n-r}
$$

Substitute the values of (4) and (5) in equation (10), we will get the pdf of $r^{\text {th }}$ order statistics $X_{(r)}$ for length biased weighted generalized uniform distribution and is given by

$$
f_{X(r)}(x)=\frac{n !}{(r-1) !(n-r) !} \frac{(\alpha+2) x^{\alpha+1}}{\beta^{\alpha+2}}\left(\left(\frac{x}{\beta}\right)^{\alpha+2}\right)^{r-1}\left(1-\left(\frac{x}{\beta}\right)^{\alpha+2}\right)^{n-r}
$$

From equation (11), the density function of largest order statistics $X_{(n)}$ is given by

$$
f_{X(n)}(x)=\frac{n(\alpha+2) x^{\alpha+1}}{\beta^{\alpha+2}}\left(\left(\frac{x}{\beta}\right)^{\alpha+2}\right)^{n-1}
$$

and the pdf of first order statistics $X_{(1)}$ is given by

$$
f_{X(1)}(x)=\frac{n(\alpha+2) x^{\alpha+1}}{\beta^{\alpha+2}}\left(1-\left(\frac{x}{\beta}\right)^{\alpha+2}\right)^{n-1}
$$

\section{LIKELIHOOD RATIO TEST}

Let $X_{1}, X_{2}, \ldots, X_{n}$ be a random sample from the length biased weighted generalized Uniform distribution. To test the hypothesis

$$
H_{0}: f(x)=f(x ; \alpha, \beta) \quad \text { against } \quad H_{1}: f(x)=f_{l}(x ; \alpha, \beta)
$$


For testing whether the random sample of size $\mathrm{n}$ comes from the generalized Uniform distribution or length biased weighted generalized Uniform distribution, the following test statistic is used

$$
\begin{aligned}
& \Delta=\frac{L_{1}}{L_{0}}=\prod_{i=1}^{n} \frac{f_{l}(x ; \alpha, \beta)}{f(x ; \alpha, \beta)} \\
& \Delta=\frac{L_{1}}{L_{0}}=\prod_{i=1}^{n}\left(\frac{(\alpha+2) x^{\alpha+1}}{\beta^{\alpha+2}} \times \frac{\beta^{\alpha+1}}{(\alpha+1) x^{\alpha}}\right) \\
& \Delta=\frac{L_{1}}{L_{0}}=\prod_{i=1}^{n}\left(\frac{(\alpha+2) x}{\beta(\alpha+1)}\right) \\
& \Delta=\frac{L_{1}}{L_{0}}=\left(\frac{(\alpha+2)}{\beta(\alpha+1)}\right)^{n} \prod_{i=1}^{n} x_{i}
\end{aligned}
$$

We reject the null hypothesis if

$$
\left(\frac{(\alpha+2)}{\beta(\alpha+1)}\right) \prod_{i=1}^{n} x_{i}>k
$$

Equivalently, we reject the null hypothesis where

$$
\Delta^{*}=\prod_{i=1}^{n} x_{i}>k^{*}, \quad \text { where } k^{*}=k\left(\frac{\beta(\alpha+1)}{(\alpha+2)}\right)^{n}>0
$$

For large sample size $n, 2 \log \Delta$ is distributed as chi-square distribution with one degree of freedom and also $p$-value is obtained from the chi-square distribution. Thus we reject the null hypothesis, when the probability value is given by $p\left(\Delta^{*}>\theta^{*}\right)$, where $\theta^{*}=\prod_{i=1}^{n} x_{i}$,

is the observed value of statistic $\Delta^{*}$.

\section{CONCLUSION}

In this paper, we have studied a new distribution called as the Length biased weighted generalized Uniform distribution. By using certain special functions, its mathematical properties, moments, failure rate, survival function has been obtained. The parameters have been estimated by using maximum likelihood method and also order statistics and fisher's information matrix have been obtained.

\section{REFERENCES}

[1] Bhatt, M. B. (2014), Characterization of Generalized Uniform Distribution through Expectation.Open Journal of Statistics, 4, 563-569.

[2] Fisher, R. A., (1934), The effects of methods of ascertainment upon the estimation of frequencies. Annals of Eugenics, 6, 13-25.

[3] Khan, M.I. and Khan, M.A.R., (2017), characterization of generalized uniform distribution based on lower record values, ProbStat Forum, 10, 23-26.

[4] Lappi J, Bailey RL., (1987), Estimation of diameter increment function or other tree relations using angle-count samples, Forest science 33: 725-739.

[5] M. Masoom Ali, Manisha Pal and Jungsoo Woo (2007), Some Exponentiated Distributions, The Korean Communications in Statistics, Vol. 14 No.1, 93-109.

[6] Para, B. A., \& Jan, T.R., (2018), On Three Parameter Weighted Pareto Type II Distribution: Properties and Applications in Medical Sciences. Applied Mathematics \& Information Sciences Letters, 6(1), 13-26.

[7] Rao, C. R., (1965), "On discrete distributions arising out of method of ascertainment, in classical and Contagious Discrete", G.P. Patiled; Pergamum Press and Statistical publishing Society, Calcutta. 320-332.

[8] Rather, A. A., and Subramanian, C., (2018), Transmuted Mukherjee-Islam failure model, Journal of Statistics Applications \& Probability, 7(2), 343-347.

[9] Rather, A. A., Subramanian, C., Shafi, S., Malik, K. A., Ahmad, P. J., Para, B. A., and Jan, T. R. (2018), "A new Size Biased Distribution with applications in Engineering and Medical Science", International Journal of Scientific Research in Mathematical and Statistical Sciences, Vol.5, Issue.4, pp.75-85.

[10] Van Deusen P.C., (1986), Fitting assumed distributions to horizontal point sample diameters. For Sci 32(1): 146-148. 